\title{
Tardive Akathisia in an Adult on Long Term Metoclopramide
}

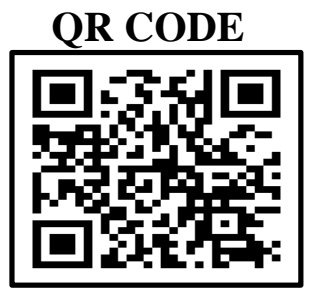

\section{SUBHSHANKAR PRASSAD'1, RON MEADOWS', NARAYAN KAMATE}

Metoclopramide is prescribed to subjects dealing with gastrointestinal issues like delayed gastric emptying, nausea, vomiting or loss of appetite. It is also used to treat chemotherapy and surgery related nausea and vomiting. Although it is very effective in targeting stomach related illnesses, severe adverse drug reactions can occur in those who take metoclopramide. This case report describes a 32-year-old female who suffered from tardive akathisia while being treated with long-term metoclopramide. Long term exposure to causative medication leads to evolution of Tardive akathisia and this can stay for a lifetime. It is important to restrict the exposure duration of triggering drug. Health care professionals and patients should be well aware of this neurological adverse event of metoclopramide.

KEYWORDS: Metoclopramide, Tardive Akathisia, Adverse Drug Reaction

\section{INTRODUCTION}

Metoclopramide is a peristaltic, antiemetic agent for short-term treatment of nausea connected with migraine, chemotherapy, radiotherapy and postoperative problems. The use of this drug is significantly more than domperidone and ondansetron. Metoclopramide is a dopamin-D2receptor antagonist, which at high concentrations also has a partial blocking and stimulating effect on serotonin 5 - $\mathrm{HT}_{3}$ and 5 - $\mathrm{HT}_{4}$-receptors. The therapeutic effect is achieved via receptors in the gastrointestinal tract, which causes increased intestinal motility, as well as via the chemoreceptor trigger zone in the central nervous system, whereby nausea is inhibited. Metoclopramide crosses the blood-brain barrier and can mediate dopamine receptor blockade in the basal ganglia with the risk of extrapyramidal side effects. ${ }^{1}$ Although majority of adverse events of this drug like headache, diarrhea, restlessness and drowsiness are relatively minor, there are several that can be medically significant like confusion, irregularity in heartbeat, difficulty breathing, difficulty swallowing, seizures, tardive akathisia and dyskinesia, depression and suicidal tendencies. This drug has the potential to cause drug-induced movement disorders, including akathisia. which is characterized by an inner restlessness resulting in a need for constant movement. We hereby report a case wherein the subject developed severe symptoms that were consistent with tardive akathisia after seven months of metoclopramide treatment.

\section{MEDICAL HISTORY}

A 32-year-old woman presented with palpitation, agitation and restlessness. She is a home maker, married and living with her husband in a metropolitan city, belonging to upper middle socio-economic status. Her medical history was significant for gall bladder removal after which she suffered from pain in the epigastric region along with other symptoms consistent with gastroesophageal reflux disease (GERD). She was given IV fluids and started with daily metoclopramide treatment giving good effect. She stayed in the hospital for a day and was discharged owing to relief in her symptoms. Except this she had no other relevant medical history, no pregnancy, no other comorbid conditions and also denied any incidence of drug allergy. After seven months of fixed treatment, however, she began feeling an inner sense of restlessness along with agitation and palpitations and stopped taking the medication on her husband's suggestion. Her symptoms worsened and her condition deteriorated significantly. She experienced constant inner restlessness, urge to move, motor turmoil and chills that occurred spontaneously or were triggered by light touch. She regarded these movements to be happening spontaneously and claimed to have no control. She was cooperative, alert and oriented in relation to time and place. She responded well to our 
questions but was a bit slow in doing this. She was anxious but showed a good general judgment. In her entire life, she had never encountered any kind of psychological or neurological issues. Also, she had never been administered metoclopramide or related drugs before gall bladder removal. She underwent a thorough investigation in the areas of neurology, endocrinology and internal medicine, but appeared to be neurological and motorically unremarkable. Objective and paraclinical examinations, including MRI scan of the cerebrum, as well as determination of the levels of plasma methanephrines, thyroidstimulating hormone and 5-hydroxyindol acetate showed nothing abnormal. Finally, the ongoing symptoms were assessed to be consistent with tardive akathisia secondary to long-term metoclopramide treatment. The subject and her husband were informed about the possible role of metoclopramide in her condition. Initially, metoclopramide treatment was stopped but there was no significant improvement in her symptoms. Later, intravenous midazolam treatment was started. Ten days later, the improvement in her anxiety level and involuntary movements was significant. Subsequently, midazolam was gradually stopped within a few days. One week after stopping midazolam, there was no recurrence of tardive akathisia symptoms. Her GERD medication has been replaced. We avoided trying metoclopramide again to avoid any kind of positive rechallenge. The subject was followed up after 1 month via a telephonic conversation with no complaints of tardive akathisia anymore.

\section{DISCUSSION}

Akathisia, which may occur as a side effect of treatment with dopamine receptor antagonists, is characterized by a feeling of inner restlessness and discomfort, as well as repetitive movements and a lack of ability to sit and stand still or unpleasant, often burning sensations in specific parts of the body. ${ }^{2}$ The condition is severely painful, but often remains undiagnosed or mistaken with anxiety/depression, because there is a lack of consensus on the diagnostic criteria. ${ }^{2,3}$ The term acute akathisia is used when the symptoms occur within days after exposure to a relevant drug and is a short-term condition that disappears upon the discontinuation of treatment. On the other hand, tardive akathisia occurs after prolonged exposure and may persist after discontinuation of the drug. Tardive akathisia belongs to the group of tardive syndromes, which also covers other movement disorders, including tardive dyskinesia and tardive tremor. ${ }^{2}$ The absolute risk of developing tardive syndromes when using metoclopramide is unknown, but in several studies an estimated risk of up to $15 \%$ has been found. ${ }^{1,3}$ Duration and dose are considered to be of great importance, just like patient-related factors such as female gender, old age, chronic diseases as well as the use of alcohol, tobacco and other neuroleptics, which increase the risk of the above. ${ }^{1-4}$ The primary action in the treatment is to discontinue the triggering substance. A slow phasing out is recommended, as sudden discontinuation may worsen or even trigger tardive syndromes. ${ }^{2}$ This could be due to the hypersensitivity of the dopamine receptors after blockade, but the pathophysiology is not fully clarified., ${ }^{1,2}$ Despite discontinuation of treatment, the symptoms may persist for years, or can be potentially lifelong. ${ }^{4}$ However, it is suggested that the condition often improves over time. The remission rate in previous studies varies greatly, between $2 \%$ and $33 \%$. Young age and early diagnosis and discontinuation of the triggering drug probably improve the prognosis. ${ }^{4}$ Tardive akathisia often does not respond to medical treatment, but treatment with tetrabenazine, which is a benzoquinolizine derivative with antihyperkinetic effect, is attempted in some persistent and disabling cases. However, the documentation of the efficacy of the above is sparse. ${ }^{2,4}$

\section{CONCLUSION}

Tardive akathisia is a disabling, often overlooked syndrome that may occur as a side effect of taking dopamine receptor antagonists. Metoclopramide should only be used for a short period of time, and special caution should be exercised when treating elderly and chronically ill patients, and the risk of neurological side effects should be kept in mind.

\section{REFERENCES}

1. Rao AS, Camilleri M. Review article: metoclopramide and tardive dyskinesia. Aliment Pharmacol Ther. 2010;31(1):11-9. $\quad$ https://doi.org/10.1111/j.13652036.2009.04189.x.

2. Fahn S, Jankovic J, Hallett M. Principles and practice of movement disorders: the tardive syndromes: phenomenology, concepts on pathophysiology and treatment, and other neuroleptic-induced syndromes. 2nd ed, Saunders, Philadelphia 2011. p.415.

3. Wijemanne S, Jankovic J, Evans RW. Movement Disorders From the Use of Metoclopramide and Other Antiemetics in the Treatment of Migraine. Headache. 2016;56(1):153-61. https://doi.org/10.1111/head.12712.

4. Zutshi D, Cloud LJ, Factor SA. Tardive Syndromes are Rarely Reversible after Discontinuing Dopamine 
Receptor Blocking Agents: Experience from a Other Hyperkinet Mov (NY). 2014 Oct 23;4:266. University-based Movement Disorder Clinic. Tremor https://doi.org/10.7916/D8MS3R8C.

\author{
Cite this article as: \\ Metoclopramide. Int Healthc \\ https://doi.org/10.26440/IHRJ/0504.07432
}

Prassad S, Meadows R, Kamate N. Tardive Akathisia in an Adult on Long Term

AUTHOR AFFILIATIONS: (*Corresponding Author)

1. MD (Internal Medicine), Consultant Medical Practioners, Kovalam, Chennai, India

2. Independent Medical Scholar, Consultant \& Researcher, Valladolid, Spain

Source of support: Nil, Conflict of interest: None declared 